\title{
A New Technique of Removing Blind Spots to Optimize Wireless Coverage in Indoor Area
}

\author{
A. W. Reza, ${ }^{1}$ K. Dimyati, ${ }^{2}$ K. A. Noordin, ${ }^{1}$ M. J. Islam, ${ }^{1}$ M. S. Sarker, ${ }^{1}$ and H. Ramiah ${ }^{1}$ \\ ${ }^{1}$ Department of Electrical Engineering, Faculty of Engineering, University of Malaya, 50603 Kuala Lumpur, Malaysia \\ ${ }^{2}$ Department of Electrical and Electronic Engineering, Faculty of Engineering, National Defence University of Malaysia, \\ 57000 Kuala Lumpur, Malaysia
}

Correspondence should be addressed to A. W. Reza; awreza98@yahoo.com

Received 6 December 2012; Revised 14 February 2013; Accepted 27 March 2013

Academic Editor: Stefano Selleri

Copyright (c) 2013 A. W. Reza et al. This is an open access article distributed under the Creative Commons Attribution License, which permits unrestricted use, distribution, and reproduction in any medium, provided the original work is properly cited.

\begin{abstract}
Blind spots (or bad sampling points) in indoor areas are the positions where no signal exists (or the signal is too weak) and the existence of a receiver within the blind spot decelerates the performance of the communication system. Therefore, it is one of the fundamental requirements to eliminate the blind spots from the indoor area and obtain the maximum coverage while designing the wireless networks. In this regard, this paper combines ray-tracing (RT), genetic algorithm (GA), depth first search (DFS), and branch-and-bound method as a new technique that guarantees the removal of blind spots and subsequently determines the optimal wireless coverage using minimum number of transmitters. The proposed system outperforms the existing techniques in terms of algorithmic complexity and demonstrates that the computation time can be reduced as high as $99 \%$ and $75 \%$, respectively, as compared to existing algorithms. Moreover, in terms of experimental analysis, the coverage prediction successfully reaches $99 \%$ and, thus, the proposed coverage model effectively guarantees the removal of blind spots.
\end{abstract}

\section{Introduction}

Recent trend shows that it is one of the greatest challenges to remove the blind spots from the user-specified environment as well as install the optimal communication system while designing the wireless networks. Blind spot [1] refers to that position where signal cannot be reached or too weak to be considered as a significant signal, which affects the overall performance of the communication system. There are not many existing methods $[2,3]$ available in the area of radio signal propagation prediction to optimize the indoor wireless coverage. In [2], a recent coverage prediction model using ray-tracing (RT) technique for field prediction, associated with genetic algorithm (GA) to optimize the base station antenna location in an indoor environment, is presented. The model is based on the image method and, thus, computational time is increased with the number of objects in the indoor environment. On the other hand, GA is used to find the location of optimal antenna that maximizes the signal strength calculated over the region of interest. The GA starts with a set of solutions, known as population, and solutions from a population are used to generate a new population. The solutions that will generate a new population are selected based on their fitness function. This procedure is repeated until a certain condition is satisfied. If the condition is not attained, genetic operators are applied to form a new population. The generation of new population completely depends on the linear combination of variables of each point in the optimization space in a certain generation. Therefore, implementation of this type of coverage prediction model is very troublesome and also computationally inefficient. Conversely, Yun's algorithm [3] also consists of GA and RT to determine the minimum number of transmitting antennas as well as their appropriate locations to provide the optimized wireless coverage for indoor environment and according to Yun et al. [3], all existing coverage models failed to guarantee the optimum wireless coverage by completely eliminating blind spots from the indoor environment. However, it may be impractical to simulate the complex environments using Yun's algorithm [3] due to expensive computational complexity that would sometimes be beyond the capacity of the contemporary computers. 
Therefore, the main research objectives are illustrated as follows: (i) to determine the best location of transmitter to provide the optimum network deployment, (ii) to determine the number of transmitters required to ensure optimal coverage, and (iii) to remove the blind spots from indoor as well as obtain the maximum wireless coverage using the minimum number of transmitters while designing the wireless networks (i.e., to find a solution where the number of transmitters is minimum and all the receivers are covered by those transmitters, which means no receiver or blind spot will exist in the indoor). To address the current challenges and prime concerns as discussed, this study exploits both RT [4-11] and GA [12-16] together with depth first search (DFS) [17] (the DFS is a last in first out searching in terms of live nodes where list of live nodes forms a stack) and branchand-bound method as a new technique that guarantees the removal of blind spots and subsequently determines the optimal wireless coverage. The RT is used here to follow the trajectory from the transmitter to the receiver. For detailed explanations of RT model, we may refer to [6]. In GA, each chromosome is represented by a binary pattern that keeps the coverage information of a transmitter. By recombination of some patterns, it finds the optimal solution. The DFS is used to search those transmitters that are required to cover all the receivers (a generated node whose child nodes have not yet been explored is called live node and the node which is being explored is called E-node [12]). While searching, the recombination theory of GA is applied to the coverage pattern of the transmitters. And, branch-and-bound method is a backtracking procedure [12], where bounding functions are applied to avoid the generation of subtrees (to avoid unnecessary searching, thus making the algorithm faster) that have no answer node. In contrast to [12], the proposed algorithm consumed less memory requirement as it stores only a stack of nodes representing a single path. If any solution is found without exploring unnecessary nodes based on the bounding functions, both space and time complexities can be reduced further. The superiority of the proposed system will be verified in the subsequent discussions.

\section{Proposed Coverage Algorithm}

The working principle of the proposed algorithm based on the DFS is as follows.

(i) Explore the root to generate its child.

(ii) Visit the child nodes and follow on with the child of them and so on until a leaf node is found.

(iii) Step back to the second child of the previous root, and so on.

For simplicity, the following notations are being used in the subsequent discussions.

(i) If the number of sampling points is $n, t_{i}$ is the $i$ th transmitter where $1 \leq i \leq n$.

(ii) The coverage pattern of a transmitter $t_{i}$ is $p_{i}$.

(iii) The number of good and bad sampling points of a coverage pattern is $N(g)$ and $N(b)$, respectively.
TABLE 1

\begin{tabular}{lcccccccc}
\hline & $e_{1}$ & $e_{2}$ & $e_{3}$ & $e_{4}$ & $e_{5}$ & $e_{6}$ & $e_{7}$ & $e_{8}$ \\
\hline$p_{x}$ & 1 & 0 & 1 & 0 & 1 & 0 & 0 & 1 \\
$p_{y}$ & 0 & 1 & 1 & 1 & 0 & 0 & 0 & 0 \\
$p_{r}=p_{x} \cup p_{y}$ & 1 & 1 & 1 & 1 & 1 & 0 & 0 & 1 \\
\hline
\end{tabular}

(iv) Let $e_{i}$ be the edge that is connected with the root and immediate child of the root node. The edge $e_{i}$ has a real-valued weight $w_{i}=\sum_{j=1}^{n} p_{i}(j) \mid p_{i}(j)=1$, where $p_{i}(j)$ is the $j$ th bit of coverage pattern $p_{i}$. Each node also has a weight of $W_{\text {node }}$. The weight of the root node is $W_{\text {node }}(0)=0=w_{0}$, the weight of the child node at level 1 is $W_{\text {node }}(1)=w_{0}+w_{1}$, and the child node at level 2 along the same path has the weight of $W_{\text {node }}(2)=w_{1}+w_{2}$. Therefore, the weight of each node can be expressed as $W_{\text {node }}(i)=\sum_{1 \leq i \leq d} w_{i-1}+w_{i}$, where $d$ and $w$ represent the depth and the weight of an edge of the tree, respectively.

In this study, the coverage information of a transmitter is described by a chromosome, which consists of a coverage pattern. The coverage pattern works as follows. Assume that an indoor area where $n$ sampling points are covered by $k$ transmitters $T=\left\{t_{1}, t_{2}, \ldots, t_{k}\right\}, k \leq n$. Here, each transmitter $t_{i}$ has a coverage pattern like $p_{i}=\left\{e_{1}, e_{2}, \ldots, e_{n}\right\}$, where $1 \leq i \leq k$ and $e_{j}=$ " 0 " or " 1 " for $1 \leq j \leq n$. The value $e_{j}=1$ indicates the $j$ th sampling point as a good sampling point, which is covered by the $i$ th transmitter and $e_{j}=0$ specifies that $j$ th sampling point is a blind spot. Again, two patterns $p_{1}$ and $p_{2}$ can generate the resultant pattern $p_{r}$ based on union operation as follows:

$$
p_{r}=\left\{p_{1} \cup p_{2} \mid \sum_{j=1}^{n} e_{j} \geq 0\right\} .
$$

In the best case, the resultant coverage pattern is

$$
p_{r}=\left\{p_{1} \cup p_{2} \cup \cdots \cup p_{k} \mid \sum_{j=1}^{n} e_{j}\right\}=n,
$$

where $e_{j}=1$ that makes summation value equal to $n$, which indicates $100 \%$ coverage. Thus, if the set of unique coverage patterns is $p=\left\{p_{1}, p_{2}, \ldots, p_{k}\right\}$, the purpose is to find a subset $p_{s} \subseteq p$, where the number of covered sampling points is

$$
\left|\bigcup_{p_{i} \in p_{s}} p_{i}\right|=n
$$

For illustration, suppose there are 8 sampling points in an indoor area. If two transmitters $t_{x}$ and $t_{y}$ cover the sampling points 1, 3, 5, 8 and 2, 3, 4, respectively, their coverage patterns $\left(p_{x}\right.$ and $\left.p_{y}\right)$ and the resultant pattern $p_{r}$ are as shown in Table 1.

Here, the resultant pattern $p_{r}$ is created based on logical "OR" operation (where the result is " 1 ", if either first or second 


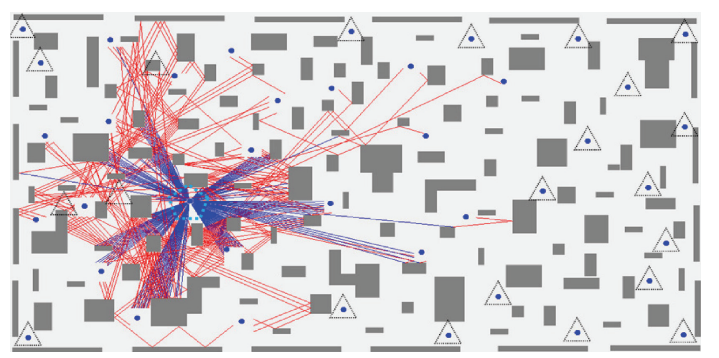

$\triangle$ One transmitting position

(a) 19 blind spots $(\Delta)$

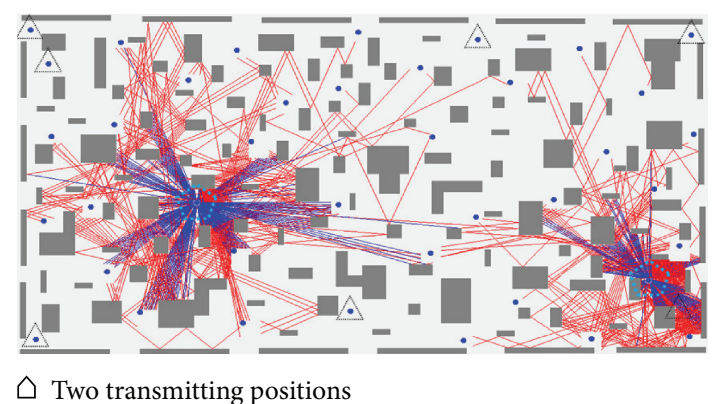

(b) 6 blind spots $(\Delta)$

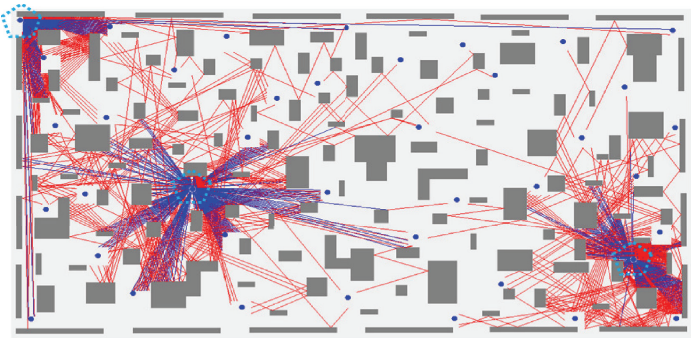

$\triangle$ Three transmitting positions

(c) No blind spot

Figure 1: Blind spots $(\Delta)$ identification and elimination from 40 sampling points deployed in an indoor environment.

bit is "1" or both bits are "1;" otherwise, the result is " 0 "). The resultant pattern $p_{r}$ consists of six good sampling points $(1,2$, $3,4,5$, and 8 ) and two blind spots (6 and 7).

Figure 1 also gives some ideas on good sampling points and blind spots, where 40 receivers have been deployed. In Figure 1(a), an area surrounded by the dotted pentagon has been chosen as the transmitter position. Here, 21 receivers (small filled circles) receive the signals from the transmitter and other 19 receivers (small filled circles surrounded by the triangle) do not receive any signal. The blue lines are the emanated rays from the transmitter, while the red lines represent the specular reflections and transmissions of waves between the objects (obstacles). Therefore, in case of a single transmitter in Figure 1(a), 21 good sampling points and 19 blind spots are found. Similarly, while using two transmitters in Figure 1(b), still there exist 6 blind spots. And, finally for Figure 1(c), where 3 transmitters are being used, there is no blind spot available that indicates that the optimal coverage for indoor wireless has been achieved and those three transmitting positions (surrounded by the dotted pentagon) are considered as at their optimized locations.

In this study, the DFS uses branch-and-bound terminology while exploring the search tree. For the indoor environment having $n$ number of sampling points, the proposed bounding functions are as follows.

(i) If the set of transmitters representing the path to the E-node is $\left\{t_{1}, t_{2}, \ldots, t_{i}\right\}$, then the set of child nodes with parent-child labeling $t_{i+1}$ is $\left\{t_{1}, t_{2}, \ldots, t_{i}, t_{i+1}\right\}$, where the coverage pattern $p_{i+1}$ is not covered by the resultant pattern $\bigcup_{1 \leq j \leq i} p_{j}$, that is, $p_{i+1} \not \subset \bigcup_{1 \leq j \leq i} p_{j}$. (ii) The number of blind spots in the resulting pattern generated from the set of transmitters until the path of the E-node is less than or equal to the summation of the good sampling points of the subsequent coverage patterns that correspond to the subtree of the root $t_{i+1}$, that is, $\left(N(b)\right.$ in $\left.\bigcup_{1 \leq j \leq i} p_{j}\right) \leq\left(N(g)\right.$ in $\left.\bigcup_{i+1 \leq j \leq n} p_{j}\right)$.

(iii) The new node will be generated from the current $\mathrm{E}$ node, if the weight $W_{\text {node }}(i)$ (where $0 \leq i \leq d$ ) of the current E-node at level $d$ is less than or equal to the number of sampling points $n$.

Consider a floor plan of a building with six sampling points as presented in [3]. It is assumed that the transmitters are placed to the slight left of the sampling points by an arbitrary distance of $36 \mathrm{~cm} \mathrm{[3].} \mathrm{The} \mathrm{reason} \mathrm{behind} \mathrm{this} \mathrm{is} \mathrm{if}$ the positions of the transmitter and receiver are identical, then the proposed algorithm will skip the corresponding sampling point while calculating the received power as both positions of transmitter and sampling points are overlapped. Now, the ray tracer for each of the six sampling positions is executed to calculate the field distribution among sampling points and the generated coverage patterns $\left(p_{1}-p_{6}\right)$ of those six transmitters $\left(t_{1}-t_{6}\right)$ are in Table 2 . Here, each row is a coverage pattern and each binary value in the column refers to a sampling point as good or blind spot for the corresponding transmitter.

From Table 2, the cost functions of the coverage patterns ( $p_{1}$ to $p_{6}$ ) are $3,3,3,4,3$, and 5 , respectively. Here, the cost function (effectiveness) of a coverage pattern is calculated based on the blind spots; that is, the lower the number of blind spots, the higher the effectiveness. It is noticed 


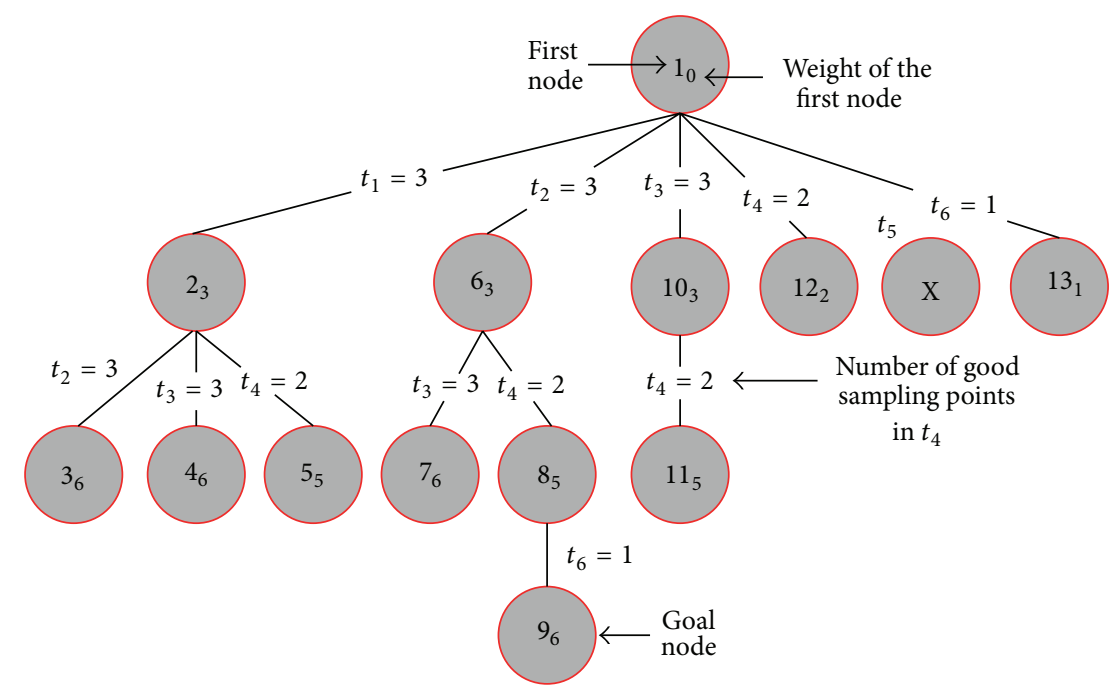

FIGURE 2: The DFS tree generated by the proposed algorithm.

TABLE 2: Coverage pattern of transmitters $\left(t_{1}\right.$ to $\left.t_{6}\right)$ in the matrix form.

\begin{tabular}{ccccccc}
\hline & $e_{1}$ & $e_{2}$ & $e_{3}$ & $e_{4}$ & $e_{5}$ & $e_{6}$ \\
\hline$p_{1}$ & 1 & 1 & 1 & 0 & 0 & 0 \\
$p_{2}$ & 0 & 1 & 1 & 0 & 1 & 0 \\
$p_{3}$ & 0 & 1 & 1 & 1 & 0 & 0 \\
$p_{4}$ & 1 & 0 & 0 & 1 & 0 & 0 \\
$p_{5}$ & 0 & 1 & 1 & 0 & 1 & 0 \\
$p_{6}$ & 0 & 0 & 0 & 0 & 0 & 1 \\
\hline
\end{tabular}

that both $p_{2}$ and $p_{5}$ are the same (i.e., the sampling points covered by $t_{5}$ have already been covered by $t_{2}$ ). Hence, $p_{5}$ is ignored marking it as a duplicate pattern. Thus, the proposed algorithm continues with 5 patterns except $p_{5}$ to select an optimal subset of them.

Figure 2 generates a DFS tree using those 5 transmitters to achieve the optimal coverage. The edge between two nodes represents a transmitter. According to the proposed algorithm, the exploration of a node is suspended as soon as a new unexplored node is generated. Then, the exploration of the new node is immediately begun.

The algorithm starts from the root node 1 at level 0 , which indicates no transmitter in the area. The root is explored and its first child node 2 is the next E-node of level 1 , where the coverage algorithm is based on single transmitter. Node 2 generates its first child 3 at level 2, which is expanded as usual to generate nodes 4 and 5 at level 3. However, it cannot be generated because of the third bounding function because the weight of each node at level 3 is greater than the sum of the number of sampling points, that is, $W_{\text {node }}(3)>n$. As the node 3 is a leaf node, the algorithm switches back to its parent node 2 . The second child node 4 of node 2 is generated and the algorithm switches to the second level again. Children of node 4 should be transmitters $t_{4}$ and $t_{6}$, respectively, but they cannot be generated because of first, second, and third bounding functions, respectively. The set of transmitters that consists of the path to the E-node 4 is $\left\{t_{1}, t_{3}\right\}$, the resultant coverage pattern of which is as follows:

$$
\begin{array}{|l|l|l|l|l|l|l|}
\hline p_{r}=p_{1} \cup p_{2} \cup p_{3} & 1 & 1 & 1 & 1 & 1 & 0 \\
\hline
\end{array}
$$

The pattern $p_{r}$ covers the pattern $p_{4}$ that violates the first bounding function. The pattern $p_{r}$ has 2 blind spots. The set of transmitters that forms the sub-tree of root $t_{6}$ is $\left\{t_{6}\right\}$. Therefore, according to the second boundary function:

$$
N(b) \text { in }\left\{t_{1}, t_{3}\right\} \leq N(g) \text { in }\left\{t_{6}\right\} \Longrightarrow 2 \leq 1 .
$$

As the condition of (5) is wrong, the algorithm will not generate the child node of $t_{6}$. And, if the node 5 is intended to add, the weight of the node 5 at level 3 exceeds the sum of the number of sampling points, that is, $W_{\text {node }}(3)>n$, which violates the third bounding function. Hence, it prevents the generation of node 5 . If the algorithm continues, the tree in Figure 2 will be constructed, where the cross (X) marked node of $t_{5}$ has duplicate coverage pattern. In Figure 2 , the optimal coverage is formed by the transmitters $t_{2}, t_{4}$, and $t_{6}$ and the solution path consists of the nodes $1,6,8$, and 9 , respectively.

Figure 3 shows some sample simulations with different numbers of sampling points generated by the proposed coverage algorithm. Here, the small filled solid circles represent the sampling points $\mathrm{Rx}$ (receiving points), the solid circles in hollow circles represent the optimized positions of the transmitters Tx that cover all the sampling points from those positions as well as eliminate all the blind spots, and the rectangles are the objects (working as obstacles).

\section{Results and Discussion}

In this section, both time and space complexities of the proposed algorithm will be derived and compared with 


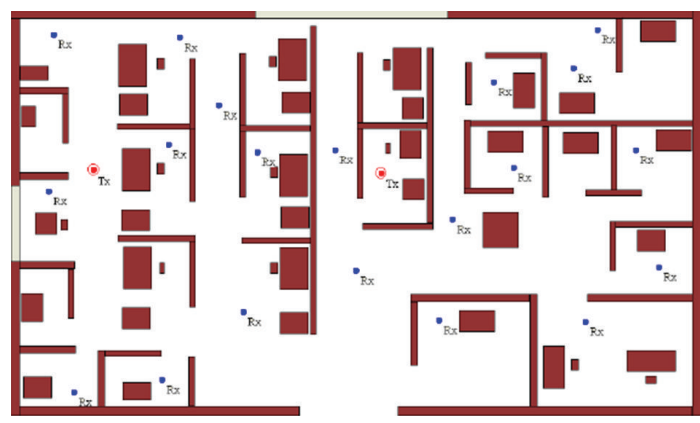

(a) 20 sampling points covered by 2 transmitters

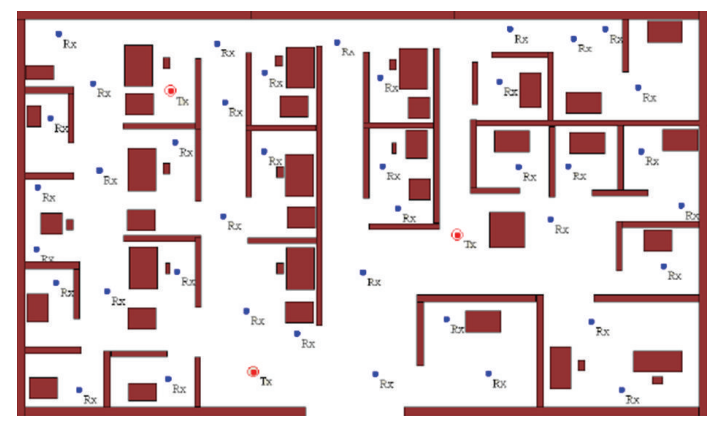

(b) 40 sampling points covered by 3 transmitters

FIGURE 3: The floor plan of the indoor propagation area and the locations of the sampling points where the optimized positions of the transmitters are surrounded by the solid circles in hollow circles.

the existing Algorithms 1 and 2, as reported in [3, 12], respectively. Both time and space complexities of the existing Algorithm 1 reported in [3] were derived in [12] and it was observed that both time and space complexities were as the same as follows:

$$
2^{n}
$$

Here, $n$ represents the number of sampling points. As the time complexity depends on the number of nodes generated or expanded until the required solution has been found, the time complexity of the existing Algorithm 2 can be expressed as follows by modifying (6):

$$
2^{n-m}-k
$$

Here, $m$ and $k$ are the number of rejected coverage patterns and unexplored nodes.

In [12], it is already proven that the time complexity is $O\left(2^{n-m}-k\right)$ according to the bounding function where BFS (breadth first search) algorithm is used. On the other hand, this paper is an enhanced version of our existing work, and hence, we can express the time complexity of DFS algorithm which is $O\left(2^{n-m}-k\right)$ as well, because the time complexity of DFS is generally the same as BFS. However, in the proposed method, we have used new bounding functions and some criteria to limit the search space tree. According to the third bounding function, the solution must be found within the path length $\left\lfloor\log _{2} n\right\rfloor+2$ for the proposed algorithm. Therefore, the improved time complexity of the proposed method is obtained as follows by replacing $n$ value with $\left\lfloor\log _{2} n\right\rfloor+2$ :

$$
2^{\left\lfloor\log _{2} n\right\rfloor+2-m}-k \text {. }
$$

As the only single path from the root to a leaf node is stored in memory stack, the space complexity of the proposed algorithm can be expressed as

$$
n-m-k^{\prime}
$$

where $k^{\prime}$ is the number of nodes unexplored in a single path. The proposed algorithm will be compared with the existing
TABLE 3: Complexities of the proposed algorithm, the existing Algorithm 1, and the existing Algorithm 2.

\begin{tabular}{lccc}
\hline Complexity & $\begin{array}{c}\text { Existing } \\
\text { Algorithm 1 }\end{array}$ & $\begin{array}{c}\text { Existing } \\
\text { Algorithm 2 }\end{array}$ & $\begin{array}{c}\text { Proposed } \\
\text { Algorithm }\end{array}$ \\
\hline Time & $2^{n}$ & $2^{n-m}-k$ & $2^{\left\lfloor\log _{2} n\right\rfloor+2-m}-k$ \\
Space & $2^{n}$ & $2^{n-m}-k$ & $n-m-k^{\prime}$ \\
\hline
\end{tabular}

Algorithm 1 [3] and the existing Algorithm 2 [12], as presented in Table 3. Based on this table, it can be deduced that the time complexity of the proposed algorithm is much better than the existing Algorithms 1 and 2 (the time complexity of both existing algorithms is increasing exponentially while it remains almost constant for the proposed algorithm). This is further demonstrated in Figure 4. In this figure, the values of $m$ and $k$ are randomly assigned to 1 and 2, respectively. That is, it has been considered that the number of rejected duplicate patterns is $m=1$ and the number of unexplored nodes is $k=2$. According to Figure 4, as the value of $m$ and $k$ increases, the complexity difference rises which indicates better performance of the proposed algorithm. In addition, Figure 5 and Table 4 show that both existing Algorithms 1 and 2 experience exponential increase in space complexity while only a linear increase with the proposed algorithm.

Let the space complexities of the existing Algorithm 1 and the proposed algorithm be $S_{1}=2^{n}$ and $S_{3}=n-m-k^{\prime}$, respectively.

Now,

$$
S_{1}-S_{3}=2^{n}-n+m+k^{\prime} \geq 0 .
$$

Thus, it can be written that $S_{1} \geq S_{3}$, which indicates less space complexity of the proposed algorithm from the existing Algorithm 1. Again suppose that the space complexities of the existing Algorithm 2 and the proposed algorithm are $S_{2}=$ $2^{n-m}-k$ and $S_{3}=n-m-k^{\prime}$, respectively.

Now,

$$
\begin{aligned}
S_{2}-S_{3} & =2^{n-m}-k-n+m+k^{\prime} \\
& =2^{n-m}-(n-m)-\left(k-k^{\prime}\right) \geq 0 .
\end{aligned}
$$




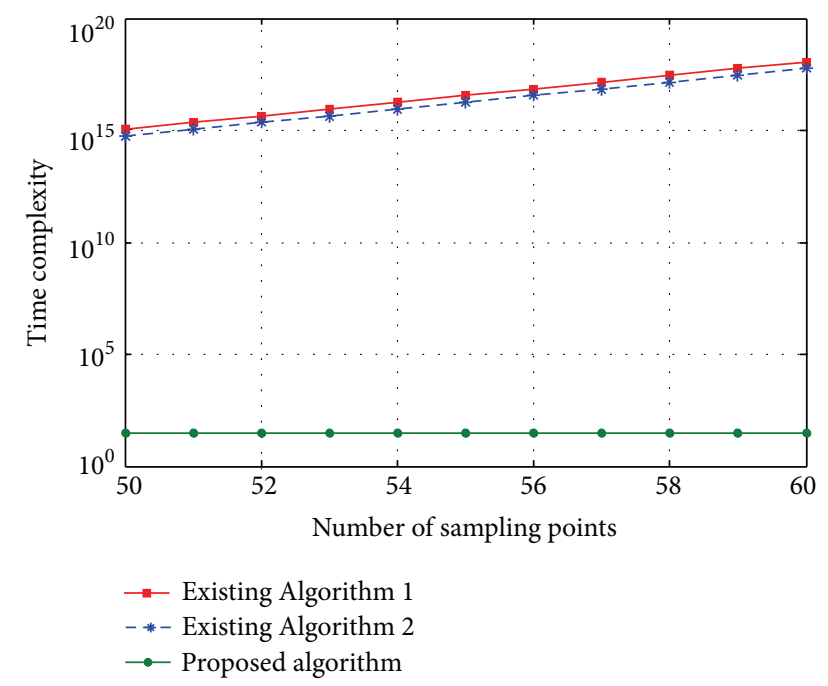

FIgURE 4: Comparison among proposed algorithm, existing Algorithm 1, and existing Algorithm 2 in terms of time complexity considering $m=1$ and $k=2$.

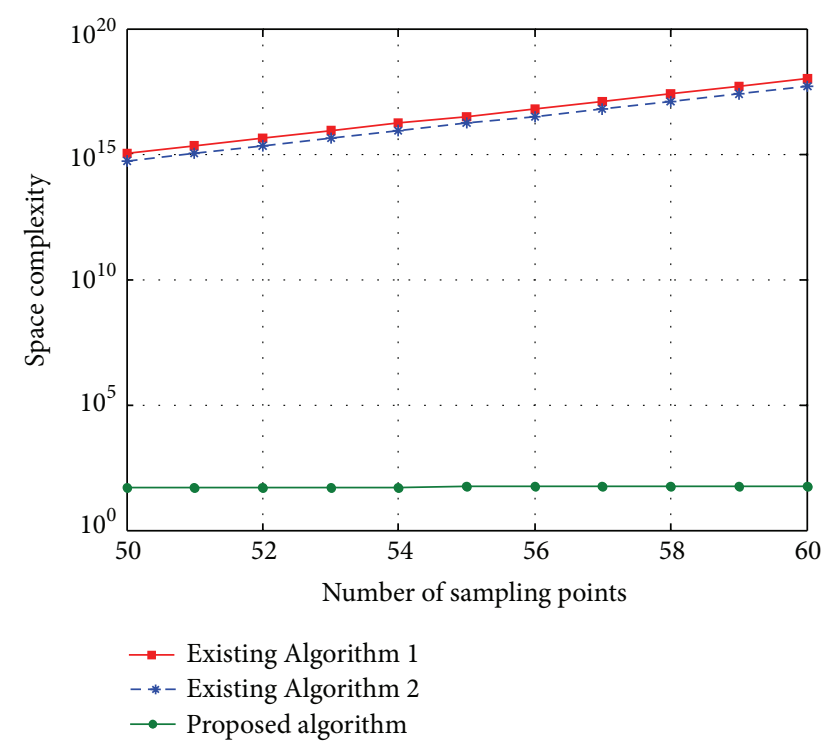

FIgURE 5: Comparison among proposed algorithm, existing Algorithm 1, and existing Algorithm 2 in terms of space complexity.

Therefore, it can also be written that $S_{2} \geq S_{3}$, which indicates less space complexity of the proposed algorithm from the existing Algorithm 2. Therefore, space complexity of the proposed algorithm is much less than that of both existing Algorithms 1 and 2 that can be best described in a tabular format as in Table 4, where the values of $m, k$, and $k^{\prime}$ are also randomly assigned to 1,2 , and 1 , respectively. That is, it has been considered that the number of rejected duplicate patterns is $m=1$, the number of unexplored nodes in the whole search space is $k=2$, and the number of unexplored nodes in a single path of the DFS tree is $k^{\prime}=1$. From Table 4 and Figure 5, it can be decided that the space complexity of both existing Algorithms 1 and 2 is increasing exponentially while the proposed algorithm is increasing linearly, which indicates an outstanding performance of the proposed algorithm.

The comparison based on the computation time among proposed algorithm and existing Algorithms 1 and 2 has been exposed in Table 5 and the reduction of computation time of the proposed algorithm from existing Algorithms 1 and 2 has been presented as a graphical view for better understanding in Figure 6. Here, 10 different scenarios containing different numbers of sampling points have been considered. The number of transmitters in Table 5 indicates how many transmitters are involved to cover all the sampling points as well as to remove all the blind spots from the corresponding propagation area. From Table 5, it is revealed that the execution time of the proposed algorithm is always much less than both existing Algorithms 1 and 2. It is also established that $75 \%$ reduction of the execution time in average is possible by the proposed algorithm while comparing with the existing Algorithm 2 and above $99 \%$ reduction (in average) is possible while comparing with the existing Algorithm 1.

In this study, specular reflections and transmissions of waves between the walls and furniture are considered, because the RT model used in this paper is a $2 \mathrm{D}$ ray tracer. Indoor measurements are performed at a carrier frequency of $2.4 \mathrm{GHz}$. The transmitter (Tx) used in this experiment is R\&S SMU200A Vector Signal Generator and the receiver $(\mathrm{Rx})$ is R\&S FSV Signal and Spectrum Analyzer. Here, the semispherical antenna is used for both of Tx and Rx to receive the signals from both directions. The obstacles are composed of arbitrary materials. The walls of the room are made of brick. The doors are made of wood and the windows are made of glass. The inside furniture of the room is made of wood and plastic board. There are some partitions that are made of plastic board and glass. Relative permittivity and refractive indexes of different materials (each and every kind of furniture in the building), such as brick, glass, wood, and plastic board in the testing room, are chosen as standard value $[18,19]$. It should be noted here that a highly sophisticated simulation software is developed using Visual Studio 2008 (C\# code) in this work that provides a Graphical User Interface (GUI). Therefore, this software will allow customizing the properties and the thicknesses of particular objects by a "mouse click" operation. In this study, all walls are assumed to have the same thickness and same properties except at locations wherever specified.

Besides, the detailed parameters, such as thickness, dielectric constant, and conductivity of particular objects, are taken for the realistic field calculations. When the properties of each object are modified, suppose that permittivity of the wall is changed, then the received signal strength will also change, because the received signal is directly related to the reflection loss, which is related to the permittivity of the materials. On the other hand, when any partition is changed by other materials, for instance, a partition made of brick is changed by the glass sheet, then the overall predicted signal is also changed, which will have an effect on the number of receivers covered by a particular transmitter, which means that the number of blind spots will also be changed. Figure 7 
TABLE 4: Comparison among existing Algorithm 1, existing Algorithm 2, and proposed algorithm in terms of space complexity considering $m=1, k=2$, and $k^{\prime}=1$.

\begin{tabular}{lccc}
\hline No. of sampling points $n$ & $\begin{array}{c}\text { Space complexity for existing } \\
\text { Algorithm 1 }\end{array}$ & $\begin{array}{c}\text { Space complexity for existing } \\
\text { Algorithm 2 }\end{array}$ & $\begin{array}{c}\text { Space complexity for proposed } \\
\text { algorithm }\end{array}$ \\
\hline 50 & $1.13 E+15$ & $5.6295 E+14$ & 48 \\
51 & $2.25 E+15$ & $1.1259 E+15$ & 49 \\
52 & $4.5 E+15$ & $2.2518 E+15$ & 50 \\
53 & $9.01 E+15$ & $4.5036 E+15$ & 51 \\
54 & $1.8 E+16$ & $9.0072 E+15$ & 52 \\
55 & $3.6 E+16$ & $1.80144 E+16$ & 53 \\
56 & $7.21 E+16$ & $3.60288 E+16$ & 54 \\
57 & $1.44 E+17$ & $7.20576 E+16$ & 55 \\
58 & $2.88 E+17$ & $1.44115 E+17$ & 56 \\
60 & $5.76 E+17$ & $2.8823 E+17$ & 57 \\
\end{tabular}

TABLE 5: Comparison among existing Algorithm 1, existing Algorithm 2, and proposed algorithm in terms of computation time.

\begin{tabular}{|c|c|c|c|c|c|c|}
\hline $\begin{array}{l}\text { No. of } \\
\text { sampling } \\
\text { points }\end{array}$ & $\begin{array}{c}\text { No. of } \\
\text { transmitters }\end{array}$ & $\begin{array}{c}\text { Time for } \\
\text { existing } \\
\text { Algorithm } 1 \\
\text { (seconds) }\end{array}$ & $\begin{array}{c}\text { Time for } \\
\text { existing } \\
\text { Algorithm } 2 \\
\text { (seconds) }\end{array}$ & $\begin{array}{l}\text { Time for } \\
\text { proposed algorithm } \\
\text { (seconds) }\end{array}$ & $\begin{array}{l}\text { Reduction from existing } \\
\text { Algorithm } 2(\%)\end{array}$ & $\begin{array}{c}\text { Reduction from existing } \\
\text { Algorithm } 1(\%)\end{array}$ \\
\hline 18 & 2 & 8.06 & 6 & 2.96 & 50.67 & 63.28 \\
\hline 20 & 2 & 30.63 & 6.75 & 2.57 & 61.93 & 91.61 \\
\hline 22 & 2 & 43.95 & 7.53 & 2.97 & 60.56 & 93.24 \\
\hline 25 & 3 & 112.02 & 7.94 & 2.35 & 70.4 & 97.9 \\
\hline 27 & 2 & 22.68 & 9.05 & 2.59 & 71.38 & 88.58 \\
\hline 30 & 3 & 183.14 & 9.31 & 2.61 & 68.59 & 98.57 \\
\hline 32 & 3 & 346.4 & 10.08 & 2.78 & 72.42 & 99.2 \\
\hline 35 & 3 & 606.95 & 10.96 & 2.83 & 74.18 & 99.23 \\
\hline 37 & 3 & 708.85 & 12 & 2.88 & 76 & 99.3 \\
\hline 40 & 3 & 292.09 & 12.22 & 2.96 & 75.78 & 98.99 \\
\hline
\end{tabular}

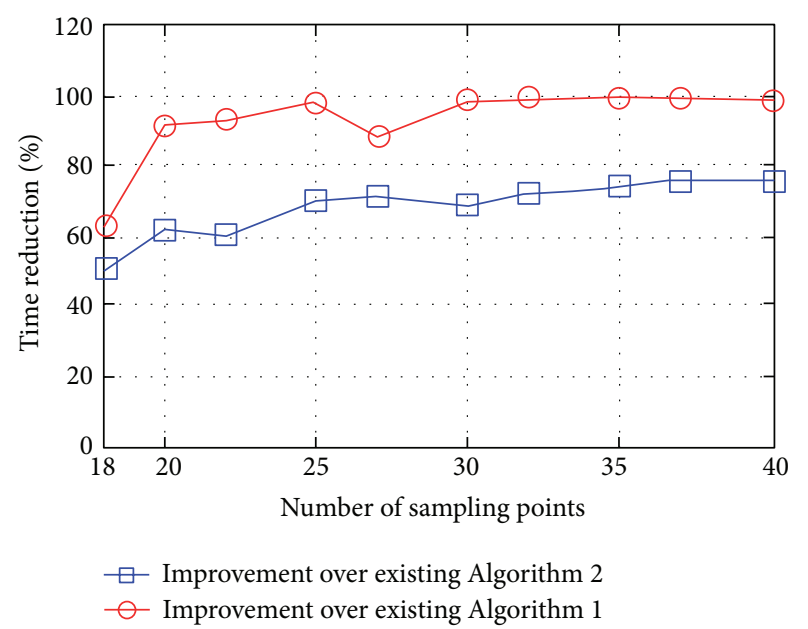

FIGURE 6: Improvement of the proposed algorithm over existing Algorithms 1 and 2. shows that the number of blind spots is changed because of changing of material used in the indoor environment.

In Figure 7(a), one transmitter and four receivers are placed in different locations. After rays are launched from the transmitter, two receivers remain uncovered which means that two blind spots exist within the coverage area, because of brick partition used in the dotted squared area. On the other hand, four receivers are covered by the transmitter when the partition walls (that exist in the dotted rectangle as shown in Figure 7(b)) are replaced by the glass sheet. Therefore, none of the blind spots exits in the coverage area. It can be summarized that when the properties of objects used in the simulation environment are changed, then the coverage pattern of the transmitter also changes.

In addition, real measurements of Figure 1 are carried out to account for the strength of the signal, because providing coverage with assessing the intensity of the signal is sufficient to claim that there are no blind spots. Bad sampling points are determined as when the received power from any transmitter 


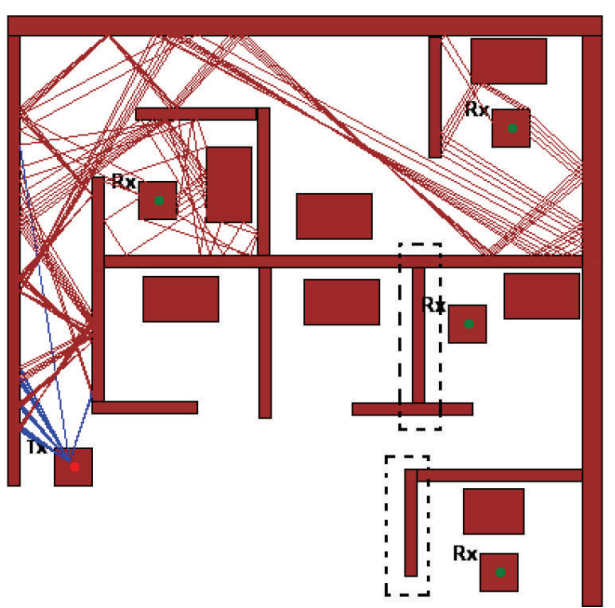

(a)

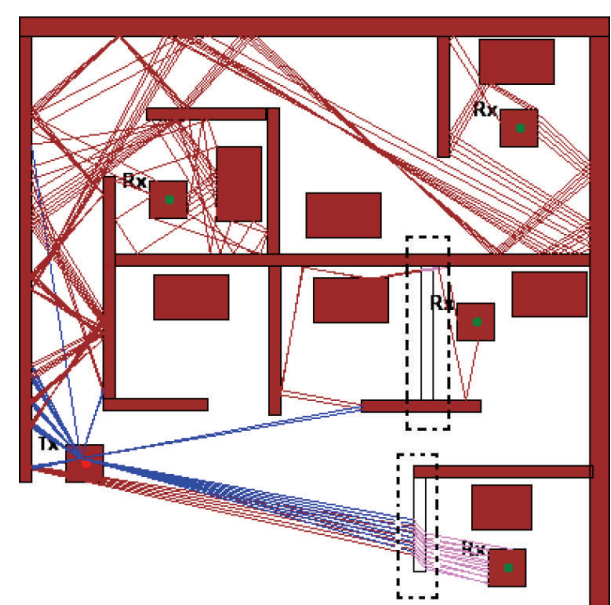

(b)

Figure 7: (a) Two blind spots exist in the coverage area when all partition walls to be considered are made of brick. (b) Blind spots are removed when some partitions are replaced by the glass sheet.

(Tx) is less than $-50 \mathrm{~dB}[3]$. Figure 8 shows a relationship between the received power and coverage for different number of $\operatorname{Tx}(\mathrm{s})$, where the transmitted power is considered as constant and the number of $\operatorname{Tx}(\mathrm{s})$ is increased to obtain the maximum coverage. From Figure 8, it is also observed that when there is a single $\mathrm{Tx}$ on the environment, it covers a less number of $\mathrm{Rx}(\mathrm{s})$. Therefore, the total power also becomes less. For two $\operatorname{Tx}(\mathrm{s})$, the number of covered $\mathrm{Rx}(\mathrm{s})$ increases, which also increases the received power. For three $\operatorname{Tx}(\mathrm{s})$, almost all $\mathrm{Rx}(\mathrm{s})$ are covered and, also, the received power is getting higher.

According to the indication of the figures, it is clear that the Rx power is coming from all of the used $\mathrm{Tx}(\mathrm{s})$. When we use single $\mathrm{Tx}$, the $\mathrm{Rx}(\mathrm{s})$ power is from that single transmitter. When there are two $\operatorname{Tx}(s)$, it is obvious that the $\operatorname{Rx}(s)$ receive power from both of the two $\operatorname{Tx}(\mathrm{s})$ and the average of the two received powers has been shown here. In case of three $\operatorname{Tx}(\mathrm{s})$, the $\operatorname{Rx}(\mathrm{s})$ receive from all three $\operatorname{Tx}(\mathrm{s})$ and here, also, the average received power is shown in the figure. During this experiment, the Tx power was kept constant at $-30 \mathrm{dBm}$. However, this experiment can also be done for any range of Tx power. From Figure 8 , it is observed that the percentage of coverage is increasing with the increase in the received power, which guarantees coverage of the receive point (i.e., there are no blind spots). The received power is also increased when the number of Txs is increasing. It is found that, when the number of Txs is 3, the percentage of coverage is almost $99 \%$ (almost all sampling points are covered).

\section{Conclusion}

An efficient and novel optimization algorithm of removing blind spots from the indoor area has been proposed in this study. The advantage of the algorithm is that the memory requirement is only linear. This is in contrast to other existing algorithms [3,12], which require more space. The reason behind that is that, a stack of nodes on the path from the root to the current node is needed to store by the algorithm. As mentioned earlier, if the proposed algorithm finds the solution without exploring unnecessary nodes in a path, the space and time it takes will be much less. Thus, it has less time and space complexities in average and the more the number of sampling points in the indoor environment, the more the complexity difference between the proposed and the existing algorithms. Thus, it is revealed that the average execution time can be reduced as high as $99 \%$ and $75 \%$, respectively, because of outstanding bounding functions as well as the concept of binary coverage pattern. Therefore, a conclusion can be drawn that the proposed algorithm excels in both algorithmic complexity and computation time.

Conversely, the wireless coverage model by [3] repeats the ray tracer for every generated chromosome. As a result, there is a high probability of repeating the RT program multiple times for the same transmitter position, which consumes a significant amount of time. The model [3] also cannot reuse the information of a transmitter location and, therefore, it runs the ray tracer repeatedly. The proposed algorithm does not run the ray tracer more than once for a single transmitting position. The proposed coverage model guarantees the removal of blind spots completely from the indoor area and subsequently continues recursively until it covers all the sampling (receiving) points. Theoretically, the coverage algorithm always covers all the receiving points to offer 100\% coverage. Moreover, the performance and accuracy of the proposed coverage model are verified by making comparisons between simulation results and measured data. It is observed that the percentage of coverage is increasing with the increase in the received power that guarantees coverage of the receiving point. The received power is also increased when the number of transmitters is increasing. Therefore, in terms of measurement results, the percentage of obtaining coverage is $99 \%$ and almost all sampling points are successfully covered in the given area. Hence, the proposed algorithm can be a great competitor of other optimization 


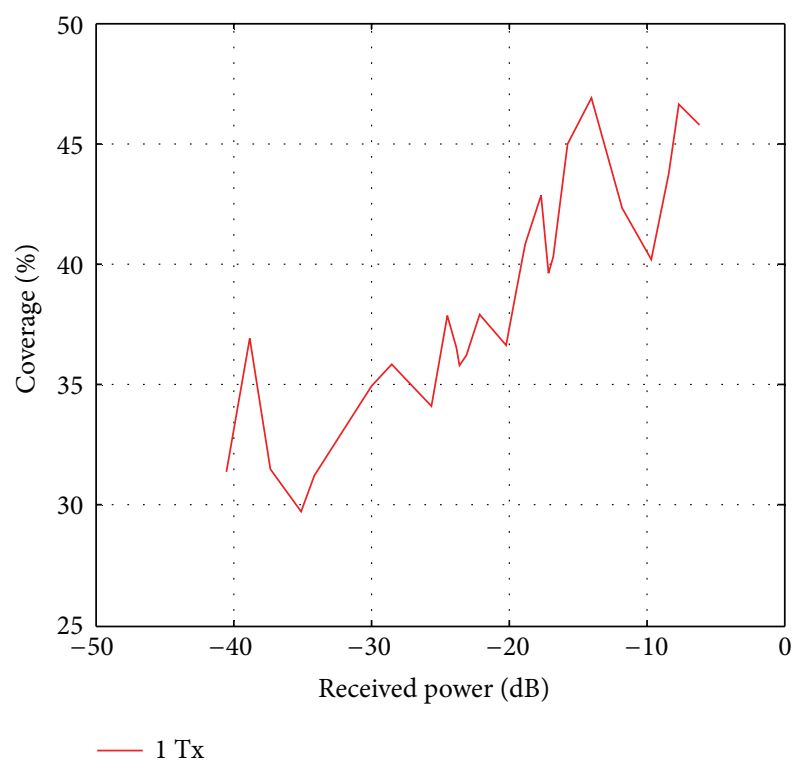

(a)

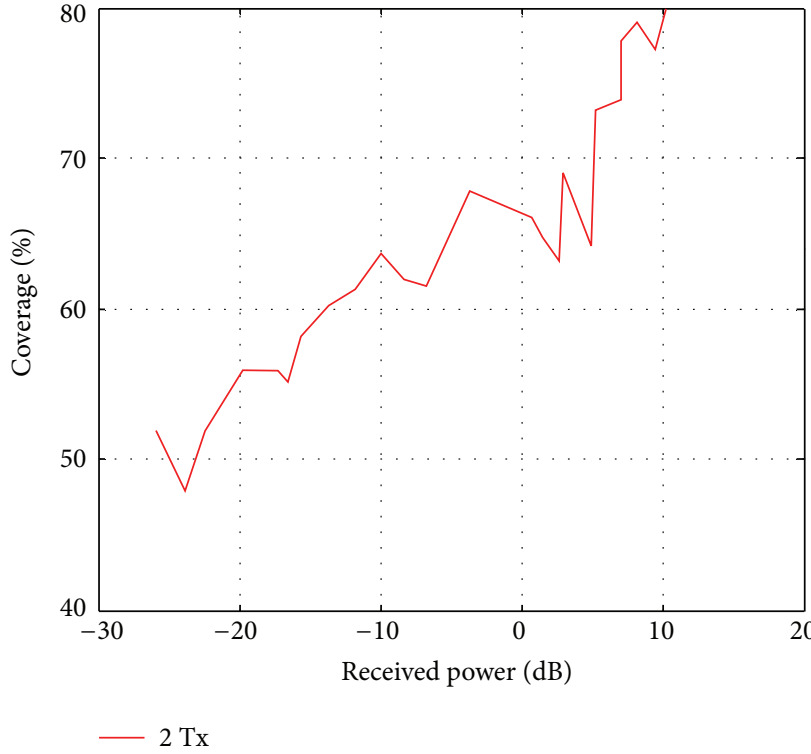

(b)

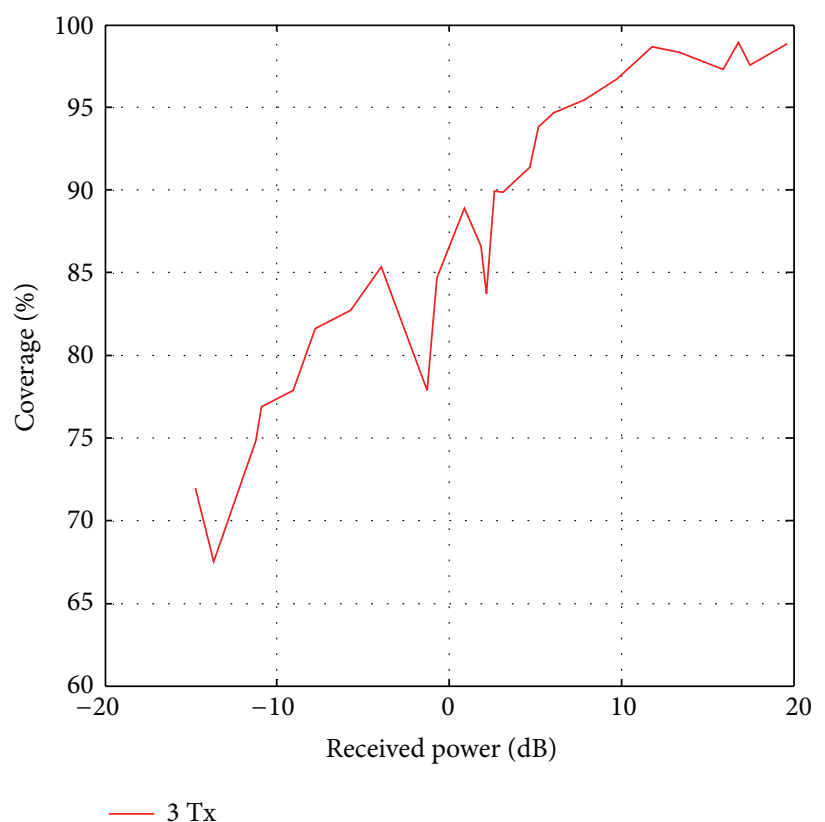

(c)

FIgURE 8: Received power versus coverage with (a) $1 \mathrm{Tx}$, (b) $2 \mathrm{Tx}$, and (c) $3 \mathrm{Tx}$.

techniques and the outcome of this study can be easily applied to real system engineering.

\section{References}

[1] C. H. Loo, A. Z. Elsherbeni, F. Yang, and D. Kajfez, "Experimental and simulation investigation of RFID blind spots," Journal of Electromagnetic Waves and Applications, vol. 23, no. 5, pp. 747$760,2009$.

[2] S. Grubisic, W. P. Carpes, and J. P. A. Bastos, "Optimization model for antenna positioning in indoor environments using 2-D ray-tracing technique associated to a real-coded genetic algorithm," IEEE Transactions on Magnetics, vol. 45, no. 3, pp. 1626-1629, 2009.

[3] Z. Yun, S. Lim, and M. F. Iskander, "An integrated method of ray tracing and genetic algorithm for optimizing coverage in indoor wireless networks," IEEE Antennas and Wireless Propagation Letters, vol. 7, pp. 145-148, 2008.

[4] M. S. Sarker, A. W. Reza, and K. Dimyati, "A novel raytracing technique for indoor radio signal prediction," Journal of Electromagnetic Waves and Applications, vol. 25, no. 8-9, pp. 1179-1190, 2011.

[5] C. Takahashi, Z. Yun, M. F. Iskander, G. Poilasne, V. Pathak, and J. Fabrega, "Propagation-prediction and site-planning software 
for wireless communication systems," IEEE Antennas and Propagation Magazine, vol. 49, no. 2, pp. 52-60, 2007.

[6] A. W. Reza, K. Dimyati, K. A. Noordin, and M. S. Sarker, "Intelligent ray-tracing: an efficient indoor ray propagation model," IEICE Electronics Express, vol. 8, no. 22, pp. 1920-1926, 2011.

[7] A. Tayebi, J. Gomez, F. Saez de Adana, and O. Gutierrez, "The application of ray-tracing to mobile localization using the direction of arrival and received signal strength in multipath indoor environments," Progress in Electromagnetics Research, vol. 91, pp. 1-15, 2009.

[8] J. Gomez, A. Tayebi, F. S. de Adana, and O. Gutierrez, "Localization approach based on ray-tracing including the effect of human shadowing," Progress In Electromagnetics Research Letters, vol. 15, pp. 1-11, 2010.

[9] Y. B. Tao, H. Lin, and H. J. Bao, "KD-tree based fast ray tracing for RCS prediction," Progress in Electromagnetics Research, vol. 81, pp. 329-341, 2008.

[10] F. Saez de Adana, O. Gutiérrez, M. A. Navarro, and A. S. Mohan, "Efficient time-domain ray-tracing technique for the analysis of ultra-wideband indoor environments including Lossy materials and multiple effects," International Journal of Antennas and Propagation, vol. 2009, Article ID 390782, 8 pages, 2009.

[11] C. Lièbe, P. Combeau, A. Gaugue et al., "Ultra-wideband indoor channel modelling using ray-tracing software for through-thewall imaging radar," International Journal of Antennas and Propagation, vol. 2010, Article ID 934602, 14 pages, 2010.

[12] A. W. Reza, M. S. Sarker, and K. Dimyati, "A novel integrated mathematical approach of ray-tracing and genetic algorithm for optimizing indoor wireless coverage," Progress in Electromagnetics Research, vol. 110, pp. 147-162, 2010.

[13] E. Agastra, G. Bellaveglia, L. Lucci et al., "Genetic algorithm optimization of high-efficiency wide-band multimodal square horns for discrete lenses," Progress in Electromagnetics Research, vol. 83, pp. 335-352, 2008.

[14] Z. Meng, "Autonomous genetic algorithm for functional optimization," Progress in Electromagnetics Research, vol. 72, pp. 253-268, 2007.

[15] Y. Rahmat-Samii and E. Michielssen, Electromagnetic Optimization by Genetic Algorithms, Wiley-Interscience, New York, NY, USA, 1999.

[16] A. W. Reza, K. Dimyati, K. A. Noordin, A. S. M. Z. Kausar, and M. S. Sarker, "A comprehensive study of optimization algorithm for wireless coverage in indoor area," Optimization Letters, 2012.

[17] D. E. Knuth, The Art of Computer Programming, vol. 1, AddisonWesley, Boston, Mass, USA, 3rd edition, 1997.

[18] D. M. Dobkin, RF Engineering for Wireless Networks: Hardware, Antennas, and Propagation, Elsevier/Newnes, Amsterdam, The Netherlands, 2005.

[19] T. S. Rappaport, Wireless Communication Principles and Practice, Communications Engineering and Emerging Technology, Prentice Hall, Upper Saddle River, NJ, USA, 2002. 

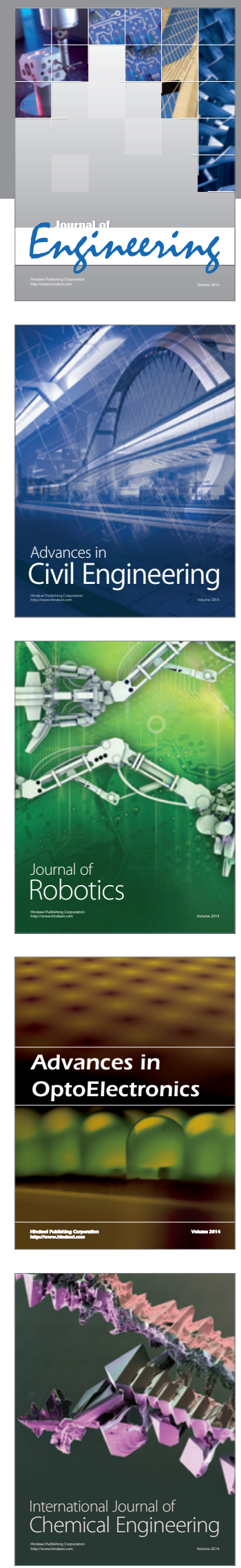

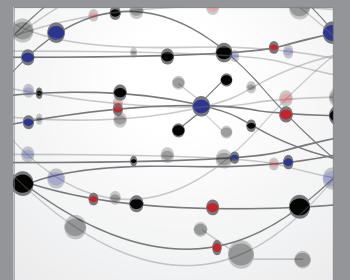

The Scientific World Journal
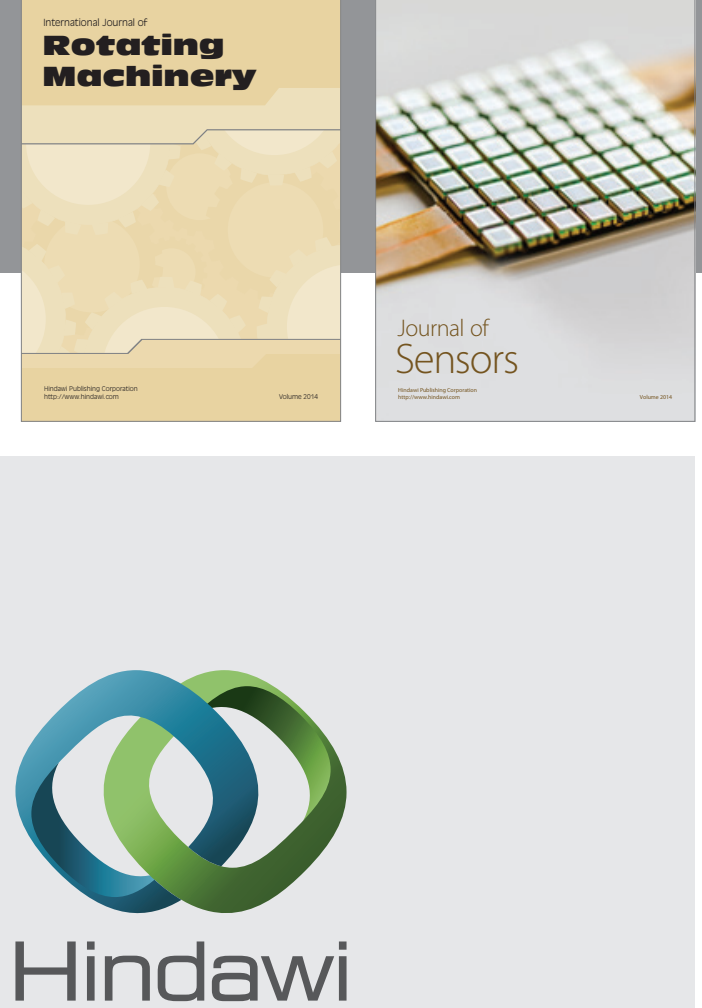

Submit your manuscripts at http://www.hindawi.com
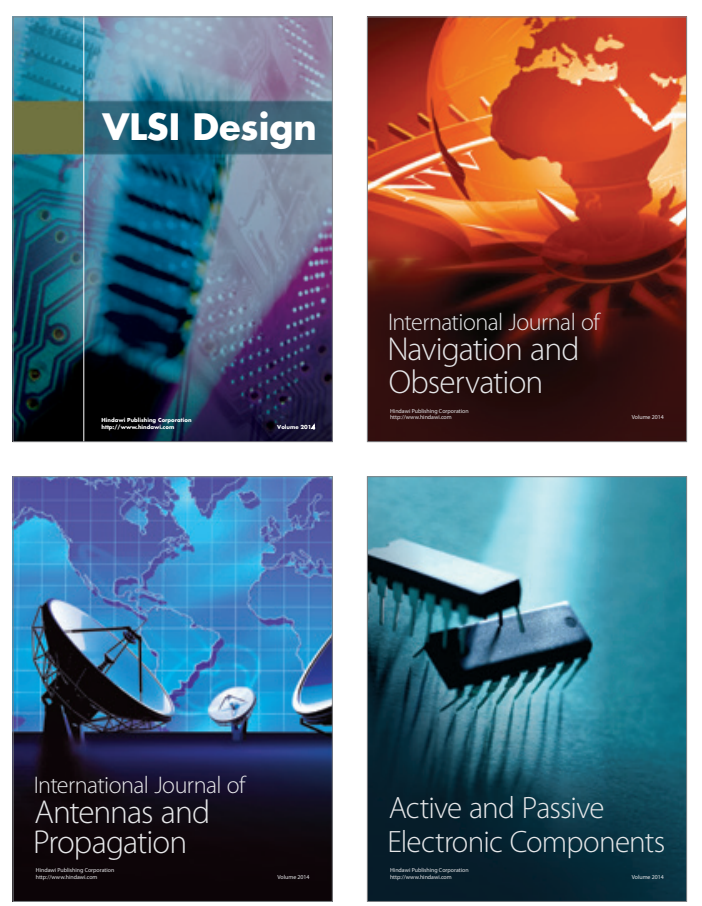
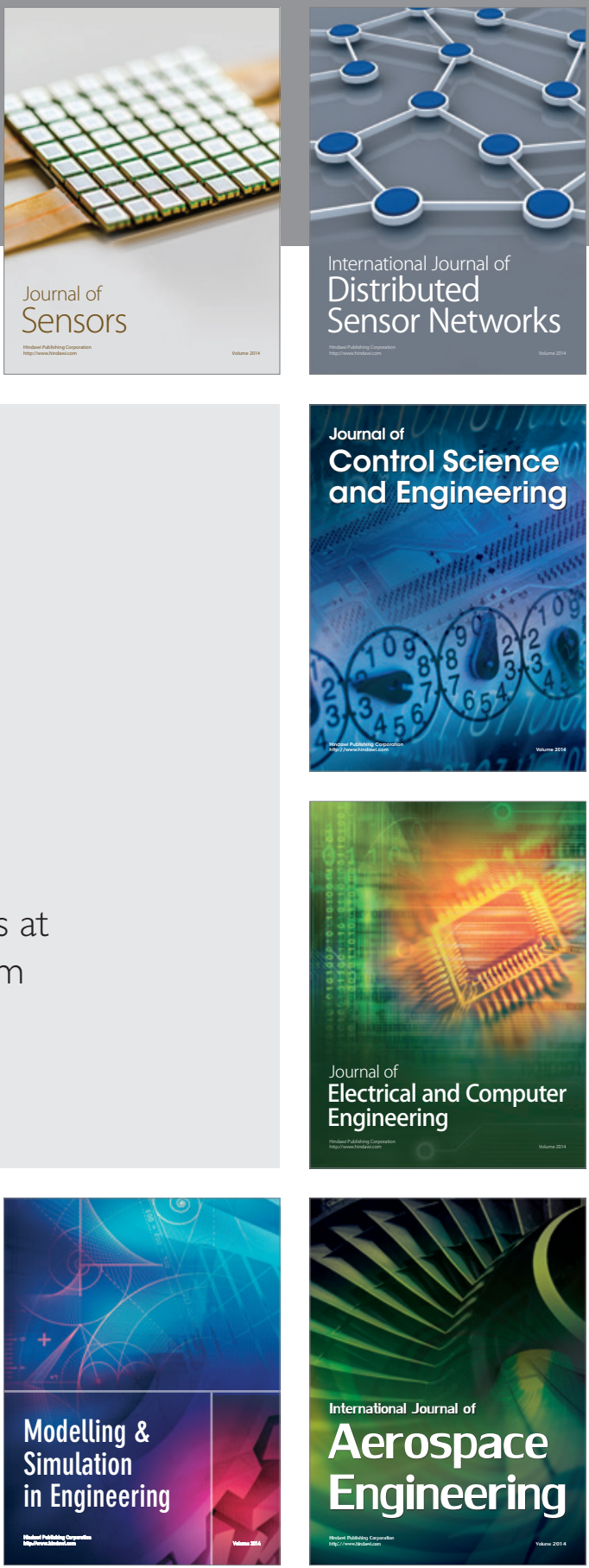

Journal of

Control Science

and Engineering
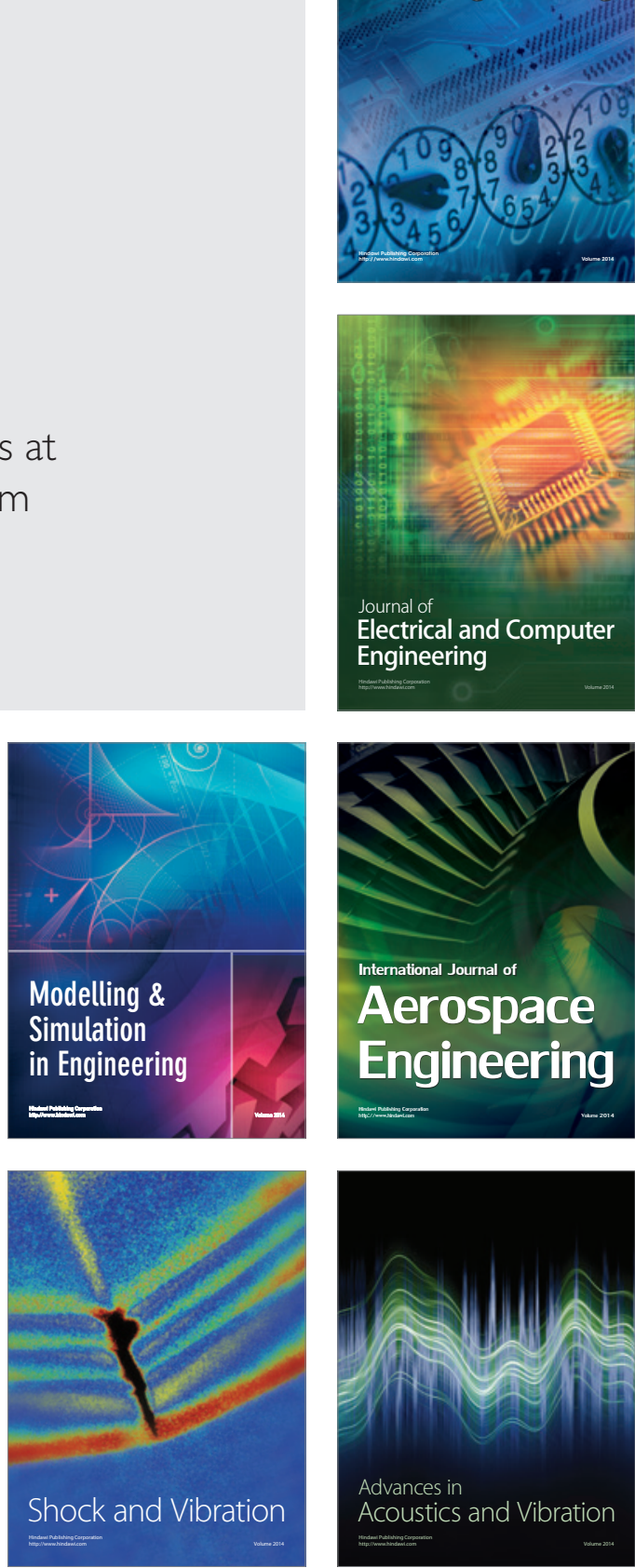